\title{
The Mystery of Two Straight Lines in Bacterial Genome Statistics
}

\author{
A.N. Gorban ${ }^{\mathrm{a}, \mathrm{c}, *}$, A.Y. Zinovyev ${ }^{\mathrm{b}, \mathrm{c}}$ \\ ${ }^{a}$ University of Leicester, Leicester, UK \\ ${ }^{b}$ Institut Curie, Paris, France \\ ${ }^{c}$ Institut des Hautes Etudes Scientifiques, Bures-sur-Yvette, France
}

Received: 9 October 2006 / Accepted: 4 May 2007 / Published online: 19 June 2007

(C) Society for Mathematical Biology 2007

\begin{abstract}
In special coordinates (codon position-specific nucleotide frequencies), bacterial genomes form two straight lines in 9-dimensional space: one line for eubacterial genomes, another for archaeal genomes. All the 348 distinct bacterial genomes available in Genbank in April 2007, belong to these lines with high accuracy. The main challenge now is to explain the observed high accuracy. The new phenomenon of complementary symmetry for codon position-specific nucleotide frequencies is observed. The results of analysis of several codon usage models are presented. We demonstrate that the mean-field approximation, which is also known as context-free, or complete independence model, or Segre variety, can serve as a reasonable approximation to the real codon usage. The first two principal components of codon usage correlate strongly with genomic $\mathrm{G}+\mathrm{C}$ content and the optimal growth temperature, respectively. The variation of codon usage along the third component is related to the curvature of the mean-field approximation. First three eigenvalues in codon usage PCA explain $59.1 \%, 7.8 \%$ and $4.7 \%$ of variation. The eubacterial and archaeal genomes codon usage is clearly distributed along two third order curves with genomic $\mathrm{G}+\mathrm{C}$ content as a parameter.
\end{abstract}

Keywords Codon usage $\cdot$ Nucleotide frequency $\cdot$ Context-free approximation · Mean field · Algebraic statistics · Principal components · Genome evolution

\section{Introduction}

The DNA double helix consists of two strands: the leading strand and the lagging strand. Pairs of complementary nucleotides, base pairs AT and GC, have different binding energies, and the GC bond is stronger. For statistical analysis of genome, a linear monotonic function of the energy of strands binding, the $\mathrm{G}+\mathrm{C}$ content (the share of GC base pairs) is used most often. Some time ago (Muto and Osawa, 1987), it was observed that the $\mathrm{G}+\mathrm{C}$ content of various parts of the genome (protein genes, stable RNA

\footnotetext{
*Corresponding author.

E-mail addresses: ag153@le.ac.uk (A.N. Gorban), Andrei.Zinovyev@curie.fr (A.Y. Zinovyev).
} 
genes, and spacers) reveals a positive linear correlation with the $\mathrm{G}+\mathrm{C}$ content of their whole genomic DNA. For coding parts, the energy of strand binding is higher than for non-coding regions. In the studies (Yeramian, 2000a, 2000b; Carlon et al., 2005), this fact was used for gene finding in a slightly more sophisticated form of "DNA melting temperature" difference: genes melt under higher temperature than intergenic regions. This approach takes into account not only the strands binding energy, but the entropy, too. It is not a miracle that the energy of strands binding defines some properties of genome, but sometimes it seems surprising that so many properties are defined by this energy. Now it has been proven that $\mathrm{G}+\mathrm{C}$ content can have a dramatic effect on the codon bias and on the amino acid composition of the encoded proteins (Lobry, 1997; Singer and Hickey, 2000).

The energy of strand binding in coding parts of genome is higher than for non-coding regions, and this difference monotonically decreases with genomic $\mathrm{G}+\mathrm{C}$ content growth (Fig. 1a, b). There are even two different effects: (i) the binding energy per base pair in a coding region is higher than in nearest non-coding regions and (ii) there exist regions in genome with typical length $10^{5}-10^{6}$ base pairs that have higher binding energy, and the share of coding part in these $\mathrm{G}+\mathrm{C}$ rich regions (i.e. concentration of genes) is higher. These effects are illustrated by Fig. 1c (see also Zinovyev et al., 2003) for several genomes.

There was a discussion, what should be chosen as the independent variable for such plots based on a simple argument: both coding and non-coding regions are parts of the genome, and it may be better to use another part, GC3, average $\mathrm{G}+\mathrm{C}$ content in the third position of codons in coding part. Because of strong correlations of $\mathrm{GC}_{i}$ (the average $\mathrm{G}+\mathrm{C}$ content in the $i$ th position of codons in coding part, $i=1,2,3)$ (Fig. 1d), in this coordinate we shall also obtain the same straight line, but with some additional noise. We prefer not to discuss coordinate choice, and follow the original work (Muto and Osawa, 1987) in data representation because of the transparent physical sense of genomic $\mathrm{G}+\mathrm{C}$ content (it is monotonic linear function of energy density). Of course, the genomic $\mathrm{G}+\mathrm{C}$ content is a symmetric characteristic, it is the same for both strands, and does not change under transposition $\mathrm{A} \leftrightarrow \mathrm{T}, \mathrm{G} \leftrightarrow \mathrm{C}$. Hence, when studying non-symmetric properties of strands, it may be useful to change the coordinate choice (Lobry and Sueoka, 2002).

In this paper, we present results of analysis of the set of known bacterial genomes (348 bacterial genomes from Genbank release April 2007). In some sense, these results are experimental ones: we analyze the experimental data from Genbank without additional ad hoc theoretical hypothesis. In special coordinates (codon position-specific nucleotide frequencies) bacterial genomes form two straight lines in 9-dimensional space: one line for eubacterial genomes, another for archaeal genomes. All the 348 bacterial genomes belong to these lines with high accuracy, and these two lines are certainly different. For lines in the codon usage space an approximation of third order has appropriate accuracy, this is a mean-field approximation (known also as complete independence model or Segre variety, Pachter and Sturmfels, 2005).

The algebraic statistics paradigm (Pachter and Sturmfels, 2005) works for this sort of data. Simple straight lines or third order curves provide unexpectedly accurate global models of data sets. We prove universality and accuracy of this phenomenon. Some slices and particular cases of these "genome trajectories" are known (Besemer and Borodovsky, 1999). They serve as important arguments in the proof of the genome code universality (Sueoka, 1962). 

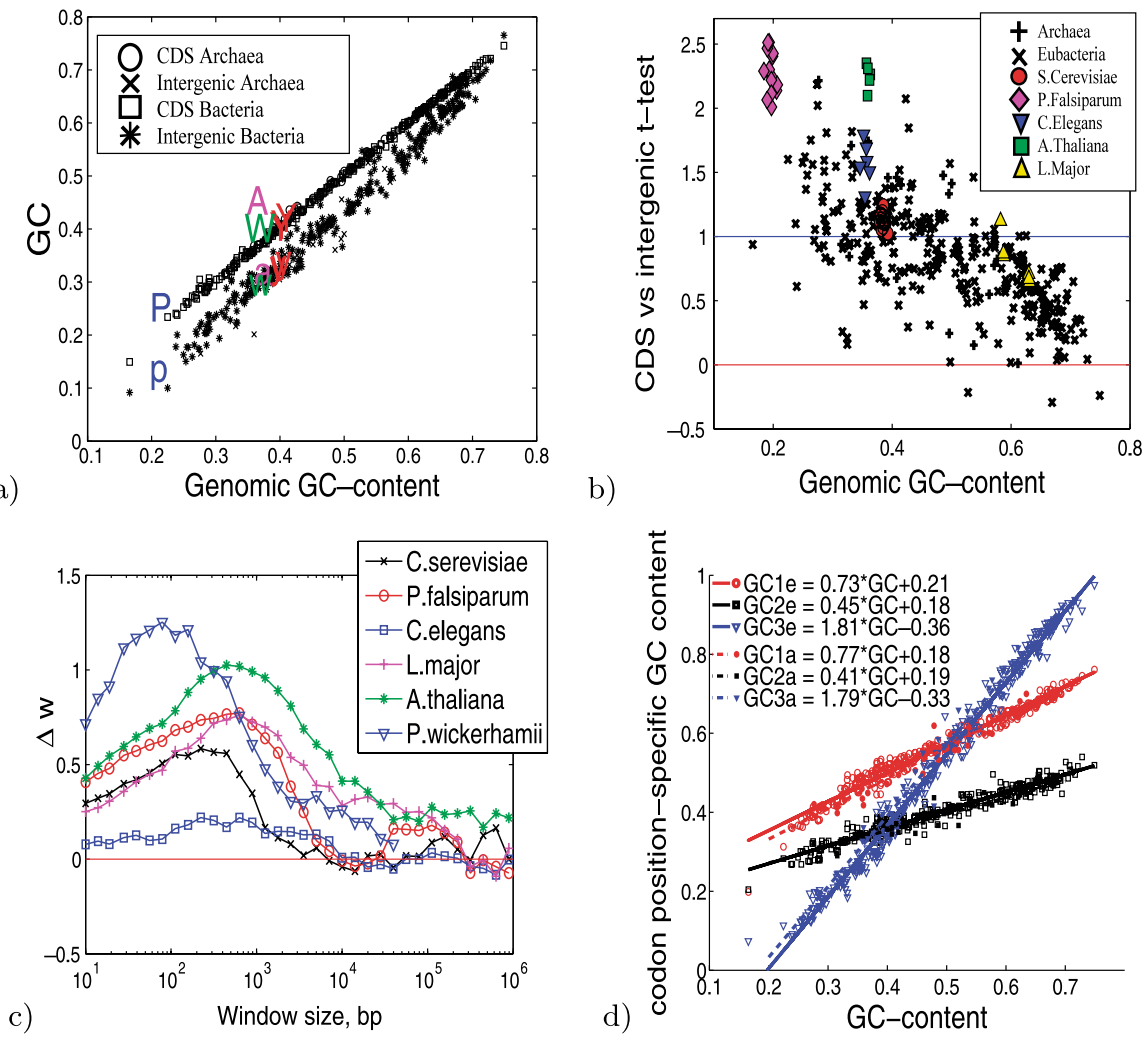

Fig. 1 (a) Average $\mathrm{G}+\mathrm{C}$ content in coding and intergenic regions as function of genomic $\mathrm{G}+\mathrm{C}$ content. On the graph 36 archaeal and 312 bacterial genomes are presented together with several chromosomes of S.cerevisiae (represented by ' $\mathrm{Y}$ ' and ' $\mathrm{y}$ ' symbols, where capital ' $\mathrm{Y}$ ' stands for $\mathrm{G}+\mathrm{C}$ content in coding regions and small ' $\mathrm{y}$ ' stands for intergenic $\mathrm{G}+\mathrm{C}$ content), A.thaliana ('A' and 'a' symbols), C.elegans ('W' and 'w' symbols) and P.falsiparum ('P' and 'p' symbols). As for S.cerevisiae, capital letters stand for $\mathrm{G}+\mathrm{C}$ content in coding regions, small letters stand for intergenic $\mathrm{G}+\mathrm{C}$ content. (b) Difference between average $\mathrm{G}+\mathrm{C}$ content in coding and intergenic regions normalized to unite variance (so-called

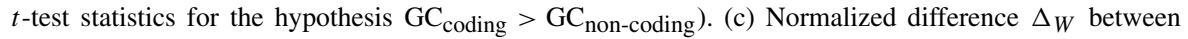
average $\mathrm{G}+\mathrm{C}$ content in windows of the length $W$ with the center belonging to coding and non-coding regions. For $i$ th base pair in the genomic sequence, the average $\mathrm{C}+\mathrm{C}$ content, $\mathrm{GC}_{W}(i)$, in the window of length $W$ centered at this base pair is calculated, $\mathrm{GC}_{W \text { cod }}$ and $\mathrm{GC}_{W \text { non-cod }}$ are average values of $\mathrm{GC}_{W}(i)$ for base pairs from coding and non-coding regions, respectively, and $\Delta_{W}$ is the normalized difference: $\Delta_{W}=\left(\mathrm{GC}_{W \text { cod }}-\mathrm{GC}_{W \text { non-cod }}\right) /\left(\operatorname{Var}\left(\mathrm{GC}_{W \text { cod }}\right)+\operatorname{Var}\left(\mathrm{GC}_{W \text { non-cod }}\right)\right)^{1 / 2}($ where Var is variance $) .(\mathrm{d})$ Position-specific $\mathrm{G}+\mathrm{C}$ content, $\mathrm{GC}_{i}$ as function of average $\mathrm{G}+\mathrm{C}$ content, $(i=1,2,3)$. Solid line and empty points correspond to 312 completed eubacterial genomes, broken line and filled symbols correspond to 36 completed archaeal genomes.

Of course, some of the linear functions appear because of simple reasons. For example, the dependence $\mathrm{CG}_{\text {cod }}\left(\mathrm{GC}_{\text {genome }}\right.$ ) (Fig. 1a) should be linear with high accuracy because of the high proportion of coding part in bacterial genomes (but even this dependency is more accurate than we could prove on the basis of this proportion). 


\section{Results}

\subsection{Two straight lines}

For each genome we can define the codon position-specific nucleotide frequencies in the coding part of this genome. The coding part of the genome is divided into codons. The codon position-specific nucleotide frequencies are 12 numbers $p_{\alpha}^{i}$, where $\alpha$ is the nucleotide symbol (A, C, G, or T), and $i=1,2,3$ is the position number of nucleotide in a codon. Among 12 frequencies $p_{\alpha}^{i}$ only 9 are independent because of the normalization conditions $p_{\mathrm{A}}^{i}+p_{\mathrm{C}}^{i}+p_{\mathrm{G}}^{i}+p_{\mathrm{T}}^{i}=1$. The possibility of non-uniqueness of separation of the coding part into codons is here neglected. The vectors with all possible coordinates $p_{\alpha}^{i}$ fill a 9-dimensional polyhedron (a direct product of three tetrahedra). In this 9-dimensional space bacterial genomes form two straight lines: one line for eubacterial genomes, another for archaeal genomes. Both of lines can be parametrized by genomic $\mathrm{G}+\mathrm{C}$ content in a very natural way, because $p_{\alpha}^{i}$ prove to be linear functions of genomic $\mathrm{G}+\mathrm{C}$ content with high accuracy. These functions are different for different lines. The results of statistical analysis (the regression lines together with experimental points) are presented in Fig. 2.

a)
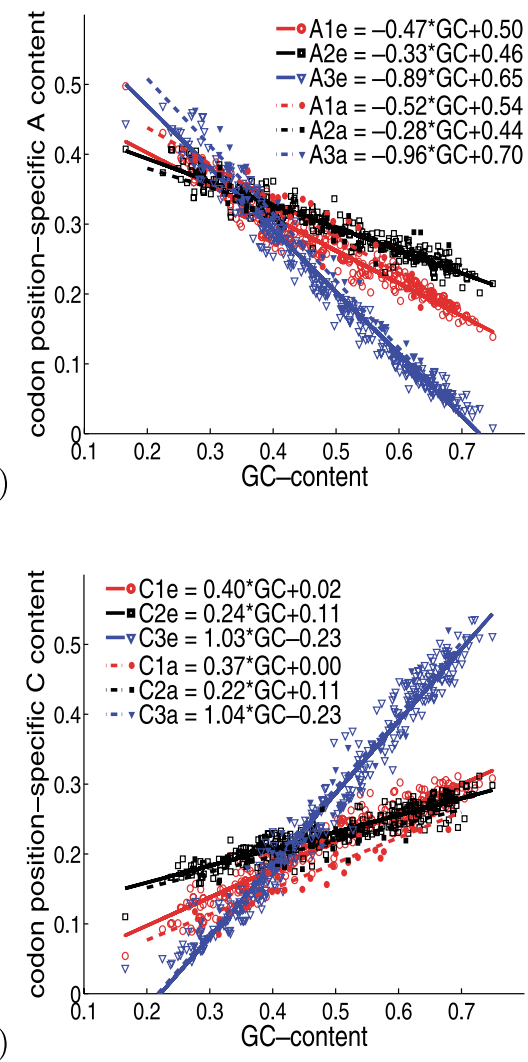

b)

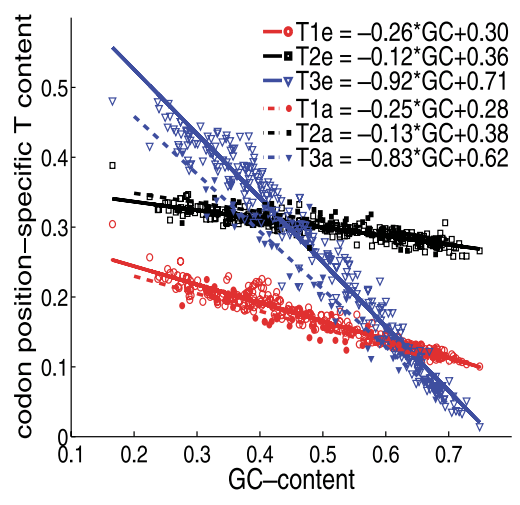

d)

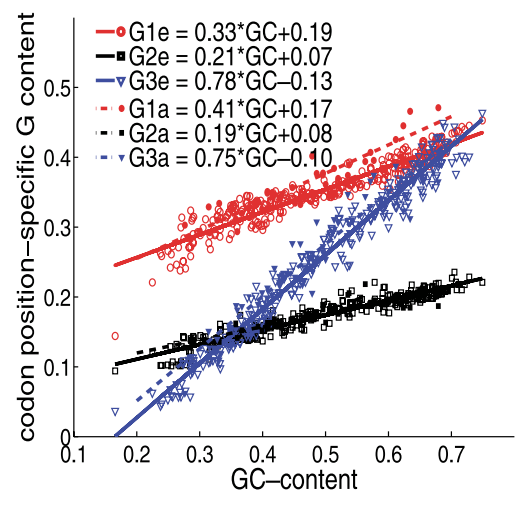

Fig. 2 Codon position-specific nucleotide frequencies as functions of average $\mathrm{G}+\mathrm{C}$ content. Solid line and empty symbols correspond to 312 completed eubacterial genomes, broken line and filled symbols correspond to 36 completed archaeal genomes. 
Table 1 Coefficients for $p_{\bullet}(\mathrm{GC})=k_{1}+k_{2} \times$ GC regression with intervals for $90 \%$ confidence level. $R^{2}$ statistics shows how much variation is explained by regression

\begin{tabular}{|c|c|c|c|c|c|c|}
\hline Function & $k_{1}$ & $k_{2}$ & $k_{1}$ interval & $k_{2}$ interval & $R^{2}$ & $p$-value \\
\hline \multicolumn{7}{|c|}{ EUBACTERIA } \\
\hline A1 & 0.496 & -0.467 & $(0.490 ; 0.501)$ & $(-0.479 ;-0.456)$ & 0.934 & $<10^{-16}$ \\
\hline $\mathrm{A} 2$ & 0.459 & -0.328 & $(0.455 ; 0.464)$ & $(-0.337 ;-0.319)$ & 0.919 & $<10^{-16}$ \\
\hline A3 & 0.647 & -0.889 & $(0.640 ; 0.654)$ & $(-0.903 ;-0.875)$ & 0.974 & $<10^{-16}$ \\
\hline $\mathrm{C} 1$ & 0.016 & 0.404 & $(0.011 ; 0.022)$ & $(0.393 ; 0.416)$ & 0.916 & $<10^{-16}$ \\
\hline $\mathrm{C} 2$ & 0.111 & 0.241 & $(0.106 ; 0.115)$ & $(0.232 ; 0.250)$ & 0.868 & $<10^{-16}$ \\
\hline $\mathrm{C} 3$ & -0.229 & 1.031 & $(-0.237 ;-0.220)$ & $(1.014 ; 1.048)$ & 0.970 & $<10^{-16}$ \\
\hline G1 & 0.191 & 0.325 & $(0.186 ; 0.197)$ & $(0.315 ; 0.336)$ & 0.892 & $<10^{-16}$ \\
\hline $\mathrm{G} 2$ & 0.069 & 0.210 & $(0.066 ; 0.072)$ & $(0.204 ; 0.217)$ & 0.904 & $<10^{-16}$ \\
\hline G3 & -0.128 & 0.777 & $(-0.135 ;-0.122)$ & $(0.765 ; 0.790)$ & 0.971 & $<10^{-16}$ \\
\hline $\mathrm{T} 1$ & 0.297 & -0.263 & $(0.293 ; 0.300)$ & $(-0.269 ;-0.256)$ & 0.930 & $<10^{-16}$ \\
\hline $\mathrm{T} 2$ & 0.361 & -0.123 & $(0.358 ; 0.364)$ & $(-0.129 ;-0.118)$ & 0.829 & $<10^{-16}$ \\
\hline $\mathrm{T} 3$ & 0.710 & -0.920 & $(0.701 ; 0.718)$ & $(-0.937 ;-0.903)$ & 0.961 & $<10^{-16}$ \\
\hline $\mathrm{GC} 1$ & 0.208 & 0.730 & $(0.202 ; 0.214)$ & $(0.718 ; 0.742)$ & 0.970 & $<10^{-16}$ \\
\hline $\mathrm{GC} 2$ & 0.180 & 0.451 & $(0.174 ; 0.185)$ & $(0.441 ; 0.462)$ & 0.941 & $<10^{-16}$ \\
\hline $\mathrm{GC} 3$ & -0.357 & 1.809 & $(-0.367 ;-0.347)$ & $(1.790 ; 1.828)$ & 0.988 & $<10^{-16}$ \\
\hline \multicolumn{7}{|c|}{ ARCHAEA } \\
\hline A1 & 0.543 & -0.522 & $(0.518 ; 0.567)$ & $(-0.574 ;-0.470)$ & 0.894 & $<10^{-16}$ \\
\hline A2 & 0.436 & -0.279 & $(0.415 ; 0.457)$ & $(-0.323 ;-0.235)$ & 0.769 & $2 \times 10^{-12}$ \\
\hline A3 & 0.701 & -0.964 & $(0.673 ; 0.730)$ & $(-1.025 ;-0.904)$ & 0.956 & $<10^{-16}$ \\
\hline $\mathrm{C} 1$ & 0.003 & 0.366 & $(-0.019 ; 0.026)$ & $(0.319 ; 0.414)$ & 0.833 & $8 \times 10^{-15}$ \\
\hline $\mathrm{C} 2$ & 0.107 & 0.224 & $(0.085 ; 0.129)$ & $(0.177 ; 0.271)$ & 0.657 & $2 \times 10^{-9}$ \\
\hline $\mathrm{C} 3$ & -0.227 & 1.042 & $(-0.262 ;-0.191)$ & $(0.968 ; 1.117)$ & 0.943 & $<10^{-16}$ \\
\hline G1 & 0.174 & 0.406 & $(0.153 ; 0.195)$ & $(0.361 ; 0.451)$ & 0.873 & $10^{-16}$ \\
\hline G2 & 0.082 & 0.189 & $(0.068 ; 0.096)$ & $(0.159 ; 0.219)$ & 0.770 & $2 \times 10^{-12}$ \\
\hline G3 & -0.098 & 0.748 & $(-0.136 ;-0.060)$ & $(0.667 ; 0.828)$ & 0.879 & $<10^{-16}$ \\
\hline $\mathrm{T} 1$ & 0.280 & -0.251 & $(0.262 ; 0.298)$ & $(-0.288 ;-0.213)$ & 0.791 & $4 \times 10^{-13}$ \\
\hline $\mathrm{T} 2$ & 0.375 & -0.134 & $(0.355 ; 0.395)$ & $(-0.176 ;-0.092)$ & 0.457 & $6 \times 10^{-6}$ \\
\hline $\mathrm{T} 3$ & 0.624 & -0.826 & $(0.589 ; 0.659)$ & $(-0.900 ;-0.751)$ & 0.912 & $<10^{-16}$ \\
\hline $\mathrm{GC} 1$ & 0.177 & 0.773 & $(0.147 ; 0.208)$ & $(0.709 ; 0.836)$ & 0.925 & $<10^{-16}$ \\
\hline $\mathrm{GC} 2$ & 0.189 & 0.413 & $(0.167 ; 0.211)$ & $(0.366 ; 0.459)$ & 0.870 & $10^{-16}$ \\
\hline GC3 & -0.325 & 1.790 & $(-0.368 ;-0.282)$ & $(1.699 ; 1.881)$ & 0.970 & $<10^{-16}$ \\
\hline
\end{tabular}

Significant differences between eubacteria and arhaea are observed for $p_{\mathrm{A}}^{1}, p_{\mathrm{A}}^{3}, p_{\mathrm{G}}^{1}$ and $p_{\mathrm{T}}^{3}$ functions (see Table 1 with intervals for $90 \%$ confidence level). Available archaeal genomes are biased towards thermophilic species and they are known to have their own specific synonymous and non-synonymous codon usage (Lobry and Chessel, 2003). This may be a possible reason for the observed difference between linear dependencies (Fig. 2) for eubacterial and archaeal genomes.

\subsection{Complementary symmetry of codon position-specific nucleotide frequencies}

There is one interesting feature of the observed dependencies on Fig. 2 which can be called phenomenon of complementary symmetry in the region around $\mathrm{GC}=50 \%$. For given position-specific frequencies $p_{\alpha}^{(i)}, i \in\{1,2,3\}, \alpha \in\{\mathrm{A}, \mathrm{C}, \mathrm{G}, \mathrm{T}\}$ let us define the 
a)

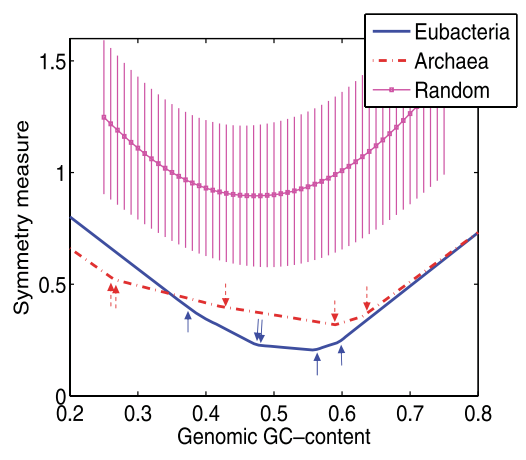

b)

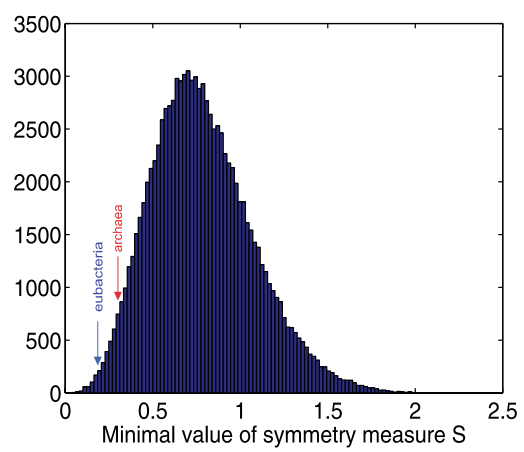

Fig. 3 (a) Complementary symmetry measure $S$ (1) as a function of GC-content. Points where the piece-wise linear curve changes its slope are shown by arrows. The curve with square markers show the average behavior of $S$ values in a random family of nucleotide frequency linear dependencies $p(\mathrm{GC})=k_{1}+k_{2} \times \mathrm{GC}$, together with standard deviations. (b) Distribution of minimal values of $S$ for the sampled family of nucleotide frequency linear dependencies. The minimal values of $S$ for eubacteria and archaea are shown by arrows.

measure of complementary symmetry $S$ :

$$
\begin{aligned}
S:= & \left|p_{\mathrm{A}}^{(1)}-p_{\mathrm{T}}^{(3)}\right|+\left|p_{\mathrm{A}}^{(2)}-p_{\mathrm{T}}^{(2)}\right|+\left|p_{\mathrm{A}}^{(3)}-p_{\mathrm{T}}^{(1)}\right| \\
& +\left|p_{\mathrm{G}}^{(1)}-p_{\mathrm{C}}^{(3)}\right|+\left|p_{\mathrm{G}}^{(2)}-p_{\mathrm{C}}^{(2)}\right|+\left|p_{\mathrm{G}}^{(3)}-p_{\mathrm{C}}^{(1)}\right| .
\end{aligned}
$$

By its meaning, $S$ is half of $l_{1}$ distance from the distribution of position-specific nucleotide frequencies to its complementary distribution (as if it would be read from the complementary strand such that $p_{\alpha}^{(i)}$ corresponds to $p_{\bar{\alpha}}^{(\bar{i})}$ where $\overline{\mathrm{A}}=\mathrm{T}, \overline{\mathrm{C}}=\mathrm{G}, \overline{\mathrm{G}}=\mathrm{C}$, $\overline{\mathrm{T}}=\mathrm{A},(\overline{1})=(3),(\overline{2})=(2),(\overline{3})=(1))$.

Let us now consider linear $p_{\alpha}^{(i)}(\mathrm{GC})$ dependencies with parameters given in Table 1 . Evidently, $S(\mathrm{GC})$ is a piece-wise linear function, changing its slope every time when any two "complementary" lines in Fig. 2 intersect. The behavior of the function is shown at Fig. 3. There are six possible slope breaks possible (since we have six terms in (1)) but only five of them happen with the positive GC-content: at $\mathrm{GC}=38.6 \%, 47.2 \%, 47.8 \%$, $55.9 \%, 59.4 \%$ for eubacteria and $\mathrm{GC}=26.4 \%, 26.6 \%, 42.0 \%, 59.0 \%, 63.0 \%$ for archaea. Qualitatively, these breaks divide GC scale in several typical regions of different "codon usage symmetry type" discussed in Gorban et al. (2005a, 2005b).

One can see from the Fig. 3 that the minimal symmetry measure $S_{\min } \approx 0.2$ is at GC $\approx$ $55.6 \%$. Is the value 0.2 big or small? Or, what is the chance that $S_{\min }$ will have such value typically and should it be close to $\mathrm{GC}=50 \%$ ? To answer this question, we considered a family of linear dependencies with $\left\{k_{1}^{p_{\alpha}^{(i)}}, k_{2}^{p_{\alpha}^{(i)}}\right\}$ parameters. The values of the parameters were limited by three requirements:

(1) Every linear dependency $p(\mathrm{GC})=k_{1}+k_{2} \times \mathrm{GC}$ should give feasible values of nucleotide frequencies (positive and less than one) at the borders of $\mathrm{GC} \in[0.25 ; 0.75]$ interval.

(2) The set of parameters should fulfill the normalization requirements $\sum_{\alpha} p_{\alpha}^{(i)}(\mathrm{GC})=1$, i.e. $\sum_{\alpha} k_{1}^{p_{\alpha}^{(i)}}=1$ and $\sum_{\alpha} k_{2}^{p_{\alpha}^{(i)}}=0$. 
(3) The set of parameters should reproduce the observed position-specific GC-content in bacteria, i.e. $k_{1}^{p_{C}^{(i)}}+k_{1}^{p_{G}^{(i)}}=k_{1}^{p_{\mathrm{GC}}^{(i)}}, k_{2}^{p_{C}^{(i)}}+k_{2}^{p_{G}^{(i)}}=k_{2}^{p_{\mathrm{GC}}^{(i)}}$, where $k_{1}^{p_{\mathrm{GC}}^{(i)}}$ and $k_{2}^{p_{\mathrm{GC}}^{(i)}}$ are read from the last three lines of the Table 1 .

We randomly and uniformly sampled 100,000 combinations of the parameters in this family. The results are presented on Fig. 3b. The expected value of $S_{\min }$ is 0.78 for this family, and the probability of reaching $S_{\min } \leq 0.2$ (eubacteria) is $0.6 \%$ and $S_{\min } \leq 0.32$ (archaea) is $3.5 \%$. The value of GC corresponding to $S_{\min }$ is found with probability $70 \%$ in the $\mathrm{GC} \in[0.35 ; 0.60]$ interval where it is distributed approximately uniformly (the data not shown). As a conclusion, the lines on Fig. 2 have rather particular combination of parameters with respect to the minimal value of complementary symmetry measure $S$.

Thus, there is another question we can ask about two lines: does evolution tune the slope of the lines on Fig. 2 such that to obtain unusually symmetric pattern of codon frequencies in the region $\mathrm{GC} \approx 50 \%$ or is it a historical trace of genome evolution? What it might be needed for? This question is a challenge for the future theoretical work.

\subsection{Validation of codon usage models}

\subsubsection{Mean-field (complete independence) model and Segre embedding}

The average codon usage ( $c u$ ) in genome is represented by 64 frequencies $p_{\alpha \beta \gamma}$, where $\alpha$, $\beta$, and $\gamma$ are nucleotide symbols, $p_{\alpha \beta \gamma}$ is the frequency of the codon $\alpha \beta \gamma$ in coding part of genome. The mean-field approximation $(m f)$ for codon usage is $p_{\alpha \beta \gamma}^{M}=p_{\alpha}^{1} p_{\beta}^{2} p_{\gamma}^{3}$. This map $\left(p_{\alpha}^{1}, p_{\beta}^{2}, p_{\gamma}^{3}\right) \mapsto p_{\alpha \beta \gamma}^{M}$ is the Segre embedding. It is widely used in algebraic geometry to consider the Cartesian product of several projective spaces as a projective variety.

The Segre embedding transforms the straight lines (Fig. 2) into lines of third order (two such lines: one for eubacterial genomes, another for archaeal genomes) in the 63dimensional simplex of codon usage (64 frequencies minus one normalization condition). The codon usage for known genomes form the clouds near these lines. These clouds have a distinctive horseshoe form (see Lobry and Chessel, 2003 and Fig. 6). If the mean-field approximation is applicable, then the clouds of codon usage are close to these lines of third order.

Analysis of the mean-field approximation accuracy was, partially, performed earlier in Knight et al. (2001). The accuracy of mean-field approximation in the 63-dimensional Euclidean space of codon usage does not mean that it is accurate in all coordinate projections uniformly. For example, it is worse for stop-codons. Violations of the mean-field approximation for usage of amino acids Arg, Val, Asp, Glu, Ser, and Cys were reported in Bharanidharan et al. (2004). In this section, we confirm some of these and other observations and perform statistical evaluation of the mean-field codon usage model for its ability to approximate real codon frequency distributions.

\subsubsection{Global approximation test}

Let us consider four statistical models of codon frequency distribution $f_{i j k}$ :

- Null background model $f_{i j k} \approx p_{i} p_{j} p_{k}$, where $p_{i}$ is simply a frequency of nucleotide $i$. This model has $4-1=3$ parameters: 4 nucleotide frequencies $p_{i}$ minus one linear relation between them, $\sum_{i} p_{i}=1$. 
Table 2 Global comparison of the statistical models of codon usage. $R^{2}$ statistics shows how much variation is explained by regression. The estimations of the regression $f_{i j k}=a+b \times f_{i j k}^{\text {model }}$ parameters are given for $\alpha=10^{-3}$ confidence level

\begin{tabular}{lllll}
\hline Model name & \# parameters & $\mathrm{a}$ & $\mathrm{b}$ & $R^{2}$ \\
\hline Null background model & 3 & $(-0.0004 ; 0.0007)$ & $(0.9617 ; 1.0241)$ & 0.33 \\
Average codon usage model & 63 (global) & $(-0.0005 ; 0.0005)$ & $(0.9700 ; 1.0300)$ & 0.35 \\
Mean-field model & 9 & $(-0.0001 ; 0.0006)$ & $(0.9675 ; 1.0005)$ & 0.633 \\
Partial independence model & 18 & $(-0.0001 ; 0.0004)$ & $(0.9756 ; 1.0009)$ & 0.748 \\
\hline
\end{tabular}

- Globally average codon usage model $f_{i j k} \approx \hat{f}_{i j k}$, where

$$
\hat{f}_{i j k}=\frac{1}{M} \sum_{i=1, \ldots, M} f_{i j k}
$$

is an average codon frequency distribution observed in all $M$ available genomes (some codons are more frequent than the others globally). This model has $63=64-1$ (global, not genome dependent) parameters.

- Mean-field or complete independence model $f_{i j k} \approx p_{i}^{1} p_{j}^{2} p_{k}^{3}$, where $p_{i}^{k}$ is the position specific frequency of the $i$ th nucleotide in the $k$ th codon position. This model has $12-3=9$ parameters: 12 position-specific nucleotide frequencies ( 4 for each position) minus 3 linear relations (1 for each position).

- Partial independence model, discussed in (Pachter and Sturmfels, 2007) $f_{i j k} \approx d_{i j} p_{k}^{3}$, where $d_{i j}$ is the frequency of the initial dinucleotide $i j$ in the codon $i j k$ (18 parameters: this model uses the full distribution of dinucleotides on the two first positions that gives $16-1=15$ parameters, and nucleotide frequencies on the third position give $4-1=3$ parameters).

We have constructed all four models for a set of bacterial genomes downloaded from GenBank, and tested the linear regression $f_{i j k}$ vs $f_{i j k}^{\text {model }}$ for the totality of all observed frequencies in 348 genomes (there are $348 \times 64=22272$ frequencies to compare). The comparison of the models is given in Table 2. It follows from the table that the mean-field approximation is twice more precise with respect to the null background distribution. The partial independence model, having twice bigger number of parameters, brings $11 \%$ of improvement in comparison to the mean-field approximation. One can conclude that the mean-field approximation can already serve as a useful (although still very simple) model of real codon frequencies.

\subsubsection{Approximation of individual genome codon distributions}

Now let us consider in some details how the mean-field approximation works. First, let us consider some bacterial genome $A$ for which we observe a codon frequency distribution $f_{i j k}^{A}$ and we approximate it by the corresponding mean-field model $m_{i j k}^{A}$. Let us also consider an alternative global average codon usage model $\hat{f}_{i j k}$, i.e. we also compare the given codon distribution to the global average calculated for all available genomes. The question is: are all the genomes approximated uniformly well? As in the previous section, we evaluate the quality of the codon usage modeling by $R^{2}$ value of the linear regression. 

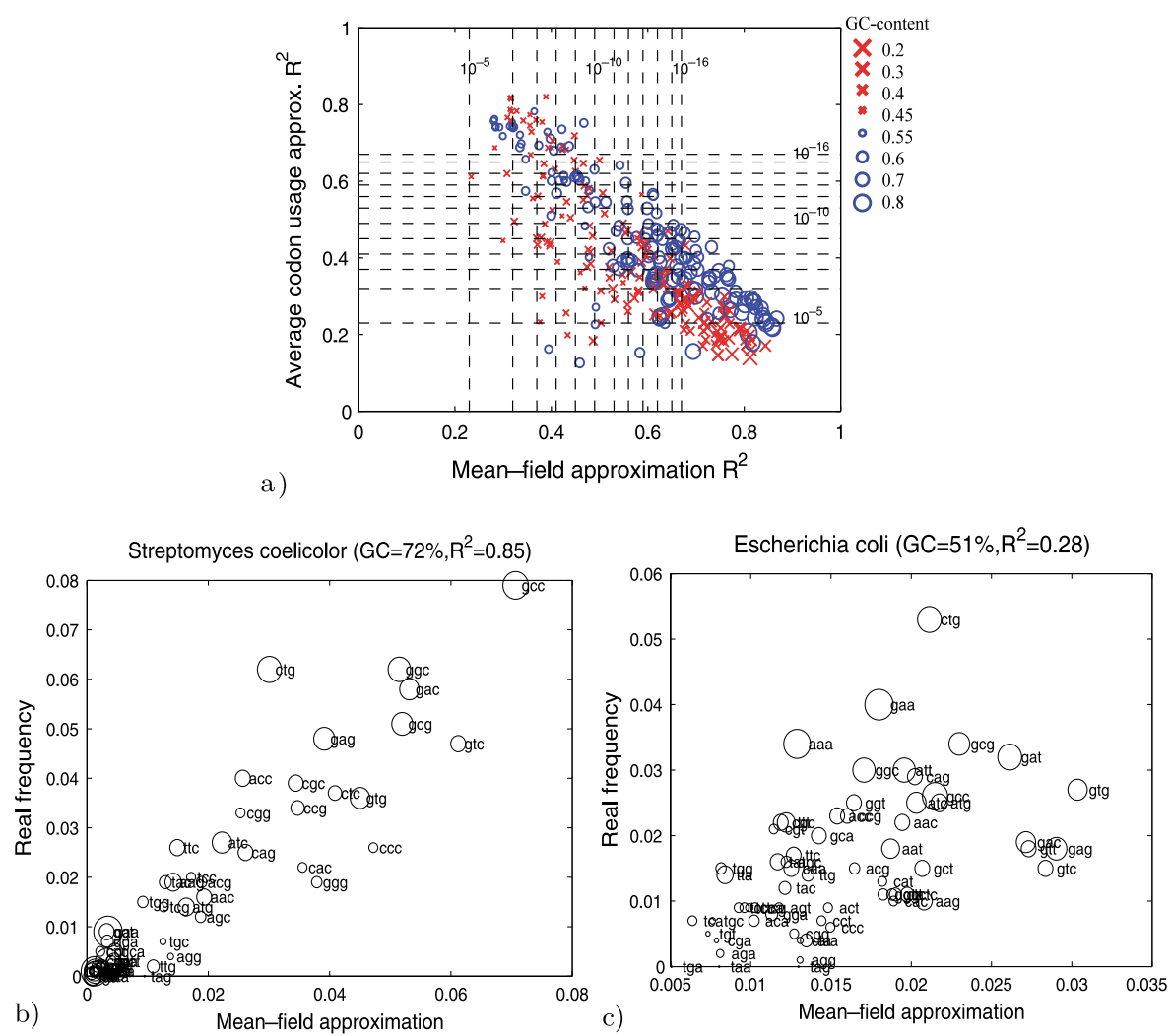

Fig. 4 (a) $R^{2}$ values for individual genomes for linear regression in two alternative codon usage models: mean-field and global average codon usage. Every point represents a genome. Circles are genomes with GC-content $>50 \%$ and the crosses correspond to GC-content $<50 \%$. Size of the point is proportional to $|\mathrm{GC}-50 \%|$ value. Dashed lines correspond to different powers of $p$-values. (b,c) Approximation of codon frequencies in the mean-field model for S.coelicolor and E.coli. Each point corresponds to a codon. The size of the point reflects global average frequency of the codon in all bacterial genomes. $R^{2}$ value is given for the mean-field model fit. It is clear that while the linear fit is worse for E.coli, its codon frequencies are well correlated with the global average codon usage $\hat{f}_{i j k}$.

On the Fig. 4a we present comparison of two alternative models $m_{i j k}$ and $\hat{f}_{i j k}$ for 348 available genomes. The resulting conclusion is quite interesting: as a rule, genomes with average GC-content close to $50 \%$ are relatively poor described by their mean-field approximations and the alternative hypothesis $f_{i j k}^{A}=\hat{f}_{i j k}$ must be accepted. For AT-rich and GC-rich genomes the mean-field approximation works significantly better than $\hat{f}_{i j k}$. One of the explanations of this observation is that close to $\mathrm{GC}=50 \%$, a genome is more free in choice of codons and this choice can be influenced by the codon bias (by evolutionary pressure) which shapes the codon distribution and can not be predicted from the position-specific nucleotide frequencies (a genome selects the most optimal codon among synonymous). However, there are genomes with GC-content close to 50\% (like 
A.marginale, T.whipplei, H.walsbyi) and whose codon usage can be well estimated from the mean-field: this means that they are less influenced by the codon bias.

There is a small group of genomes for which neither mean-field nor average approximation work well (see Fig. 4a): the most typical are H.butylicus, A.pernix, M.thermoautotrophicum, P.abyssi, P.torridus, T.acidophilum, A.aeolicus, M.thermophila, T.pendens, W.succinogenes, T.kodakaraensis. Interestingly, most of them are archaeal habitating in extremal conditions ( 4 of them belong to Thermoprotei, 2 to Thermococci, 2 to Thermoplasmata families, also one in Methanobacteria and one in Methanomicrobia). Apparently, the environment of these bacteria greatly influences their codon composition and makes them different from the common trends.

On Fig. 4b, two typical examples of genomes are presented, one is well described by the mean-field approximation and the other better fits the global average codon usage model.

\subsubsection{Approximation of individual codon frequencies}

Now let us consider an individual codon $I K J$ and look how its value is approximated by the mean-field model through all 348 bacterial genomes. In Fig. 5 the results of the linear regressions are given for 64 codons. It is clear from the figure that three usual stop codons tag, tga , taa, non-standard stop codon agg, standard start codon atg and non-standard ttg as well as cga codon (which can be easily mutated to tga via a methylation/deamination mechanism) can not be well approximated by the mean-field model. There is some tendency that more frequent codons are better approximated (for example, relatively rare codons $c c c$ and $g g g$ are much worse approximated than more frequent $a a a$ and $t t t)$. Note that the regression slope in the fit is not always close to 1 although it is distributed for various codons around mean value 1 with standard deviation about 0.35 . This reflects the fact that along some directions of codon frequency space, projection onto the mean-field manifold leads to distortions, such as systematic under- or overestimated codon frequen-

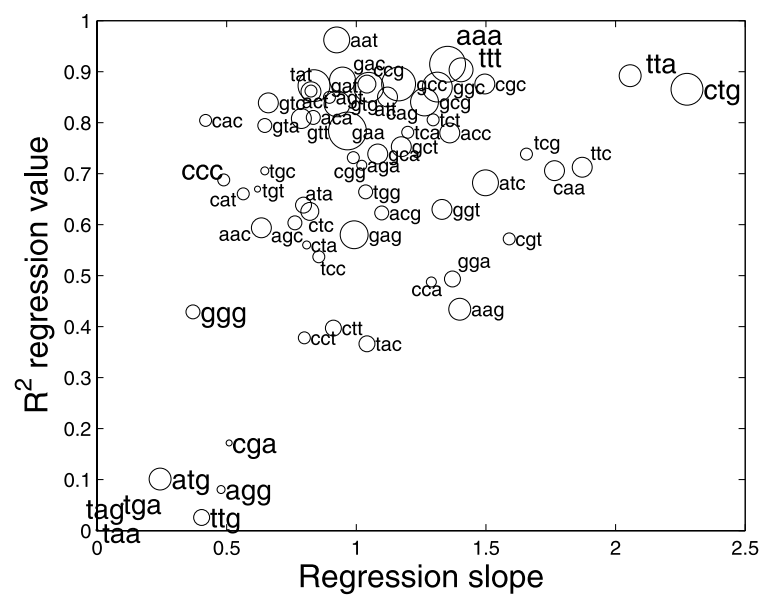

Fig. $5 R^{2}$ values versus regression slope (parameter $b$ in $f_{I J K}^{i}=a+b \times m_{I J K}^{i}$, where the regression is estimated over all observed genomes $i=1.348$ for a fixed codon $I J K$ ). Each point corresponds to a codon. The size of the point reflects average frequency of the codon in all bacterial genomes. 
cies. For example, the frequency of $g g g$ codon is systematically over-estimated on the 9-dimensional mean-field manifold while frequency of two alternative start codons $\mathrm{ctg}$ and $t t a$ is systematically under-estimated.

\subsection{PCA analysis of bacterial codon usage and the mean-field model}

On Fig. 6, we show 3D PCA plot ${ }^{1}$ visualizing genome average codon usage distributions.
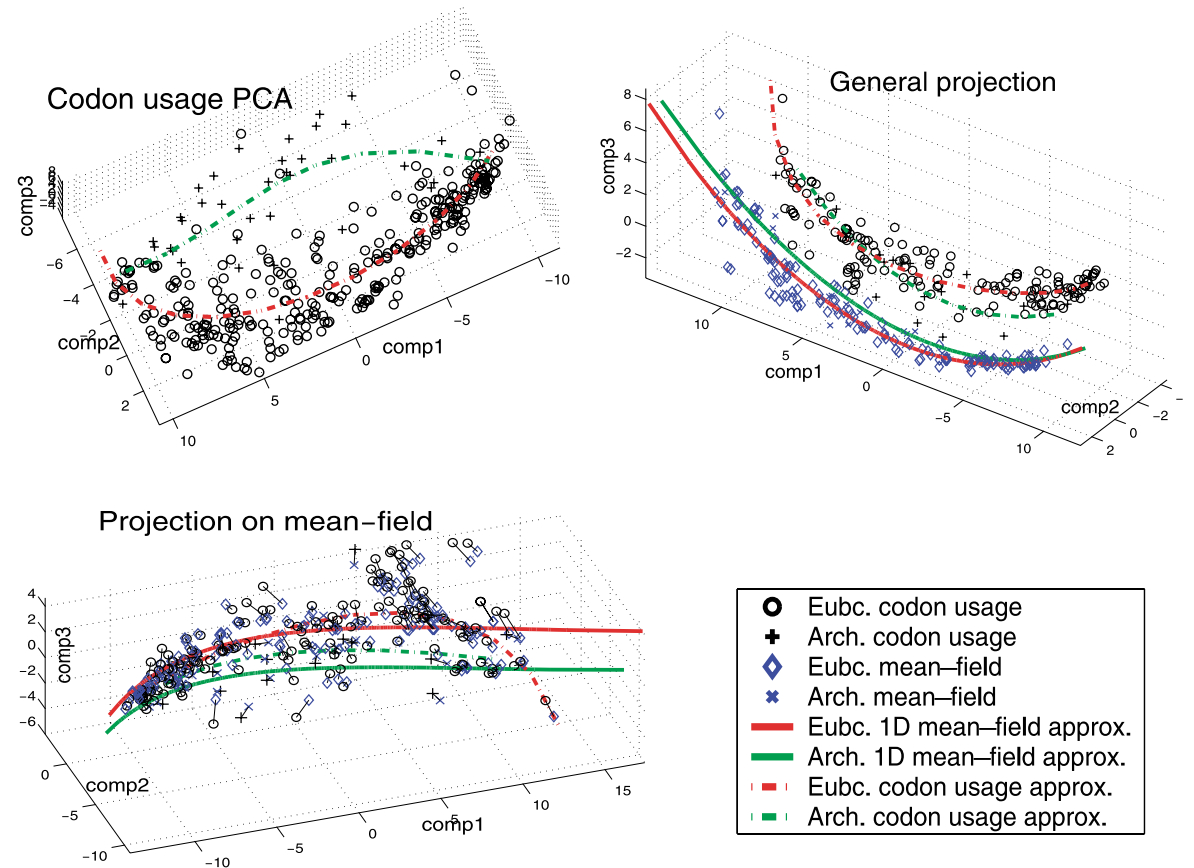

\begin{tabular}{|ll|}
\hline - & Eubc. codon usage \\
+ & Arch. codon usage \\
Eubc. mean-field \\
$*$ & Arch. mean-field \\
& Eubc. 1D mean-field approx. \\
- Arch. 1D mean-field approx. \\
- Eubc. codon usage approx. \\
- Arch. codon usage approx.
\end{tabular}

Fig. 6 PCA plots for the average codon usage and the corresponding mean-field approximations for 348 bacterial genomes; top-left: PCA plot of the average codon usage; top-right: projection on the linear envelope of principal components calculated for the average codon usage and the corresponding mean-field approximations taken together; bottom-left: projection on the linear envelope of principal components calculated for the mean-field approximations only. Solid lines represent triplet frequencies calculated with use of one-dimensional approximation of mean-field approximation (see Fig. 2), stroked lines represent fitting distribution of the average codon usage using third order curves. On the projection onto mean-field (bottom-left), same genomes represented by two points (codon usage and its mean-field approximation) are shown connected. The lengths of the lines correspond to the distance to the mean-field manifold.

\footnotetext{
${ }^{1}$ The basic PCA procedure is quite simple. The $q$-dimensional PCA plane gives the best approximation of data in the following sense. For a finite sample, $\left\{x_{i}\right\}$ the mean point $y_{0}=\sum_{i} x_{i} / n$ minimizes the sum of Euclidean distance squares $\sum_{i}\left(x_{i}-y_{0}\right)^{2}$. The first principal component vector, $y_{1}$, minimizes the sum of distance squares from points $x_{i}$ to a straight line $\left\{y_{0}+\alpha y_{1} \mid \alpha \in R\right\}$, the second principal component vector, $y_{2}$, is orthogonal to $y_{1}$ and minimizes the sum of distance squares from points $f_{i}$ to a plane $\left\{y_{0}+\alpha_{1} y_{1}+y_{2} \psi_{2} \mid \alpha_{1,2} \in R\right\}$, and so on. Vectors of principal components $y_{i}$ are the eigenvectors of the sample covariance matrix $\Sigma$. Presented PCA plot is the projection onto 3D space spanned by the three first principal component vectors.
} 
First three eigenvalues in codon usage PCA explain $59.1 \%, 7.8 \%$ and $4.7 \%$ of variation. To estimate the number of principal components we should retain (effective data dimension) we apply broken-stick distribution test (Cangelosi and Goriely, 2007). First values in the broken-stick distribution for 64 dimensions are $7.3 \%, 5.8 \%$ and $5.0 \%$. This means that formally this test gives dimension 2 , however, the third values in both distributions are really close, thus, it is reasonable to consider 3-dimensional PCA.

The first two principal components (coordinates in PCA basis) have been shown (in Lynn et al., 2002; Chen et al., 2004) to correlate strongly with genomic G+C content and the optimal growth temperature or context-dependent nucleotide bias, respectively. The variation of codon usage along the third component was not discussed in the literature, but from Fig. 6 it could be extracted that it is related to the curvature of the mean-field approximation manifold $\boldsymbol{M}$. The eubacterial and archaeal genomes are clearly distributed along two trajectories and we approximated them by fitting third order curves with genomic $\mathrm{G}+\mathrm{C}$ content as a parameter:

$$
p_{\alpha \beta \gamma} \approx a_{\alpha \beta \gamma} \mathrm{GC}^{3}+b_{\alpha \beta \gamma} \mathrm{GC}^{2}+c_{\alpha \beta \gamma} \mathrm{GC}+d_{\alpha \beta \gamma},
$$

where coefficients $a_{\alpha \beta \gamma}, b_{\alpha \beta \gamma}, c_{\alpha \beta \gamma}, d_{\alpha \beta \gamma}$ are fitted from data separately for eubacterial and archaeal genomes. The order three is chosen because the mean-field approximations are known to be distributed along trajectories of the third order $\left(p_{\alpha \beta \gamma}^{M}=\right.$ $p_{\alpha}^{1}(\mathrm{GC}) p_{\beta}^{2}(\mathrm{GC}) p_{\gamma}^{3}(\mathrm{GC})$, where all dependencies on GC are close to linear, see Fig. 2). These trajectories are also shown on Fig. 6 (top-right, bottom-left). On Fig. 6 (bottomleft), we use PCA to project both the average codon usage $c u$ and the mean-field approximations $m f$ onto the linear principal 3D manifold calculated for the mean-field approximations only. In this projection ${ }^{2}$ one can see that, indeed, the mean-field approximation works quite nicely, but on the other linear manifold, constructed for the totality of the points, one can see that the mean-field approximations have a very particular displacement (see Fig. 6 (top-right)). This observation makes the story with mean-field approximation far from being completely trivial: one has to explain why vectors connecting $c u$ and $m f$ points are almost co-linear on this picture and the $m f$ points are collected together: Is this simply an artefact of projection or there is a specific direction of information loss in the 64-dimensional space? At least it is clear that the average codon usage is not simply randomly dispersed in the vicinity of $\boldsymbol{M}$, but its ring-like spatial structure is somehow specifically oriented relative to the mean-field approximation manifold.

\section{Discussion}

We demonstrate that in 9-dimensional space of position-specific nucleotide usage $p_{\alpha}^{i}$ bacterial genomes form two straight lines: one line for eubacterial genomes, another for archaeal genomes. Both of lines can be parametrized by genomic $\mathrm{G}+\mathrm{C}$ content in a very natural way, because $p_{\alpha}^{i}$ prove to be linear functions of genomic $\mathrm{G}+\mathrm{C}$ content with high accuracy. These functions are different for different lines. The new phenomenon of complementary symmetry for codon position-specific nucleotide frequencies is observed,

\footnotetext{
${ }^{2}$ In fact, this 3D manifold is almost perfectly embedded into the $\boldsymbol{M}$ manifold. This means that this is a principal "view" from within $\boldsymbol{M}$.
} 
which also needs theoretical explanation. All these observations are statistically verified and do not depend on any codon usage model. These simple and beautiful facts can serve as good benchmarks for testing the mutation-selection-evolution models.

We show that the eubacterial and archaeal genomes in the 63-dimensional (64-1) space of codon usage are distributed along two trajectories that are third order curves parametrized by genomic $\mathrm{G}+\mathrm{C}$ content, and in the 9-dimensional space (12-3) of codon position-specific nucleotide frequencies bacterial genomes form two straight lines: one line for eubacterial genomes, the other for archaeal genomes. Some hints to observed structure were reported recently in studies on multivariate analysis of bacterial codon usage (for example, see Fig. 6 from Lobry and Chessel (2003), or in codon bias study in Carbone et al. (2005)). Some other illustrations can be found in our publications (Gorban and Zinovyev, 2004; Gorban et al., 2005a, 2005b) and on the web-site (Cluster structures in genomic word frequency distributions, 2004).

Our observations are consistent with previous studies (Sueoka's neutrality plots, etc. Sueoka, 1988; Wan et al., 2004). Nevertheless, the accuracy of the linear approximations (Fig. 2) seems to be surprising. The correlation of amino-acids usage with genomic $\mathrm{G}+\mathrm{C}$ content was studied previously for 59 bacterial genomes (Lobry, 1997). It is not difficult now to build a selection-mutation model that produces straight lines of position-specific codon usage, just following the Sueoka theory of directional mutation pressure and neutral molecular evolution (Sueoka, 1988). These lines appear as quasi-steady states of a system of the first order reactions for a given concentration of one component (the genomic $\mathrm{G}+\mathrm{C}$ content). The main challenge is to explain the observed high accuracy.

Codon usage is linked causally to a wide variety of both adaptive and non-adaptive factors including tRNA abundance, gene expression level, rates and patterns of mutations, protein structure, etc. (Knight et al., 2001) How do we explain the accuracy of Fig. 2 and of Table 1 quantitatively? This challenging question already stimulates new hypotheses and studies of algebraic structures in genetic code and genomes (Frappat and Sciarrino, 2006), and new mutation models are invented also (Minichini and Sciarrino, 2006).

There exists hierarchy of statistical models of codon usage (Pachter and Sturmfels, 2007): from the null background distribution (3 parameters), to the mean-field (or contextfree or complete independence model) approximation (9 parameters) and the partial independence model (18 parameters). We tested these three models. Of course, the highest model in the tested hierarchy, the partial independence model, gives the best results, but the mean-field model is only $11 \%$ less precise (see Table 2 ) and has twice less parameters. We can conclude that the mean-field model can serve as a reasonable approximation to the real codon usage. How to explain the accuracy of the simple mean-field approximation (Table 2 and Fig. 6) quantitatively? We still do not know an answer to this question.

\section{Acknowledgements}

We are grateful to M. Gromov (IHES) and to participants of workshop "Geometry of Genome" (Leicester, August 2005) for stimulating discussion. 


\section{References}

Besemer, J., Borodovsky, M., 1999. Heuristic approach to deriving models for gene finding. Nucleic Acids Res. 27(19), 3911-3920.

Bharanidharan, D., Bhargavi, G.R., Uthanumallian, K., Gautham, N., 2004. Correlations between nucleotide frequencies and amino acid composition in 115 bacterial species. Biochem. Biophys. Res. Commun. 315, 1097-1103.

Cangelosi, R., Goriely, A., 2007. Component retention in principal component analysis with application to cDNA microarray data. Biol. Direct 2, 2, doi:10.1186/1745-6150-2-2

Carbone, A., Kepes, F., Zinovyev, A., 2005. Codon bias signatures, organisation of microorganisms in codon space and lifestyle. Mol. Biol. Evol. 22, 547-561.

Carlon, E., Malki, M.L., Blossey, R., 2005. Exons, introns, and DNA thermodynamics. Phys. Rev. Lett. 94, 178101.

Chen, S.L., Lee, W., Hottes, A.K., Shapiro, L., McAdams, H.H., 2004. Codon usage between genomes is constrained by genome-wide mutational processes. PNAS 101(10), 3480-3485.

Cluster structures in genomic word frequency distributions, 2004. Web-site: http://www.ihes.fr/ / zinovyev/7clusters

Frappat, L., Sciarrino, A., 2006. Conspiracy in bacterial genomes. Physica A 369, 699-713.

Gorban, A.N., Zinovyev, A.Y., 2004. The mystery of two straight lines in bacterial genome statistics. arXiv q-bio.GN/0412015

Gorban, A.N., Zinovyev, A.Y., Popova, T.G., 2005a. Four basic symmetry types in the universal 7cluster structure of 143 complete bacterial genomic sequences. In Silico Biol. 5, 0025. On-line: http://www.bioinfo.de/isb/2005/05/0025/

Gorban, A., Popova, T., Zinovyev, A., 2005b. Codon usage trajectories and 7-cluster structure of 143 complete bacterial genomic sequences. Physica A 353, 365-387.

Knight, R.D., Freeland, S.J., Landweber, L.F., 2001. A simple model based on mutation and selection explains trends in codon and amino-acid usage and GC composition within and across genomes. Genome Biol. 2, 0010.1-0010.13

Lobry, J., 1997. Influence of genomic G+C content on average amino-acid composition of proteins from 59 bacterial species. Gene 205(1-2), 309-316.

Lobry, J.R., Sueoka, N., 2002. Asymmetric directional mutation pressures in bacteria. Genome Biol. 3(10), 0058 .

Lobry, J.R., Chessel, D., 2003. Internal correspondence analysis of codon and amino-acid usage in thermophilic bacteria. J. Appl. Genet. 44(2), 235-261.

Lynn, D.J., Gregory, A.C., Singer, G.A.C., Hickey, D.A., 2002. Synonymous codon usage is subject to selection in thermophilic bacteria. Nucleic Acids Res. 30(19), 4272-4277.

Minichini, C., Sciarrino, A., 2006. Mutation model for nucleotide sequences based on crystal basis. Biosystems 84, 191-206, arXiv q-bio.BM/0506010

Muto, A., Osawa, S., 1987. The guanine and cytosine content of genomic DNA and bacterial evolution. Proc. Natl. Acad. Sci. USA 84, 166-169.

Pachter, L., Sturmfels, B. (Eds.), 2005. Algebraic Statistics for Computational Biology. Cambridge University Press, Cambridge.

Pachter, L., Sturmfels, B., 2007. The mathematics of phylogenomics. SIAM Rev. 49(1), 3-31.

Singer, G.A.C., Hickey, D.A., 2000. Nucleotide bias causes a genomewide bias in the amino acid composition of proteins. Mol. Biol. Evol. 17, 1581-1588.

Sueoka, N., 1962. On the genetic basis of variation and heterogeneity of DNA base composition. Proc. Natl. Acad. Sci. USA 48, 582-592.

Sueoka, N., 1988. Directional mutation pressure and neutral molecular evolution. Proc. Natl. Acad. Sci. USA 85(8), 2653-2657.

Wan, X.F., Xu, D., Kleinhofs, A., Zhou, J., 2004. Quantitative relationship between synonymous codon usage bias and GC composition across unicellular genomes. BMC Evol. Biol. 4(1), 19.

Yeramian, E., 2000a. Genes and the physics of the DNA double-helix. Gene 255, 139-150.

Yeramian, E., 2000b. The physics of DNA and the annotation of the Plasmodium falsiparum genome. Gene $255,151-168$.

Zinovyev, A.Y., Gorban, A.N., Popova, T.G., 2003. Self-organizing approach for automated gene identification. Open Syst. Inf. Dyn. 10, 321-333. 